\title{
LA CHIMICA E I BENI CULTURALI, UN CONNUBIO VINCENTE: STUDIO TECNOLOGICO DI CERAMICHE E VETRI ATTRAVERSO I SECOLI
}

\author{
PAOLA FERMO (*) \\ Nota presentata dal m.e. Stefano Maiorana \\ (Adunanza dell's ottobre 2015)
}

SuNTO. - Nel campo delle ricerche inerenti la caratterizzazione, la conservazione ed il restauro dei beni culturali, nonché le tecniche di fabbricazione degli oggetti, è fondamentale l'impiego di metodi diagnostici che spesso vanno sotto il nome di tecniche archeometriche. L'archeometria (che misura ciò ch'è antico) si occupa dello studio scientifico con analisi di laboratorio dei materiali di cui i beni di interesse storico, archeologico, artistico e architettonico sono costituiti. Nell'ambito dello studio dei manufatti ceramici particolarmente interessante è il caso delle ceramiche rinascimentali italiane decorate con la tecnica del lustro, un film metallico iridescente di colore oro o rosso rubino ottenuto a partire da argento e da rame in forma nano-particellare mediante una tecnica molto sofisticata che testimonia le conoscenze estremamente avanzate di cui erano in possesso gli antichi vasai.

$* * *$

ABSTRACT. - In the field of the researches concerning the characterization, conservation and restoration of cultural heritage, as well as the objects manufacturing techniques, it is essential to use diagnostic methods that are referred to as archaeometric techniques. Archaeometry (which measures what is ancient) deals with the scientific study, carried out by laboratory analyses, of ancient materials and objects with the aim to deep the knowledge of historical, archaeological, artistic and technological aspects. In the field of the study of ceramic artefacts, the case of Italian Renaissance ceramics decorated with the luster technique is particularly interesting; luster is an iridescent metallic film of gold or ruby red color obtained from silver and copper in the form nanoparticles using a very sophisticated technique that testifies to the extremely advanced knowledge possessed by the ancient potters.

(*) Dipartimento di Chimica, Università degli Studi di Milano, Italy.

E-mail: paola.fermo@unimi.it 


\section{INTRODUZIONE}

La chimica, ed in particolare la chimica analitica, è di grande supporto alla studio dei beni culturali in quanto permette di acquisire informazioni importantissime inerenti le tecniche impiegate per la realizzazione di manufatti lapidei, pittorici, ceramici, vitrei, metallici, ecc. Generalmente le indagini diagnostiche su un manufatto partono dalla individuazione delle materie prime impiegate nella sua realizzazione ovvero: quale tipo di marmo è stato impiegato nella costruzione di una monumento o per la realizzazione di una statua? Quali pigmenti sono stati utilizzati dall'artista in una certa opera pittorica? Quali argille sono state impiegate dagli antichi vasai per ottenere un certo impasto ceramico? La chimica è in grado di fornire una valida risposta a tutti questi quesiti e permette in molti casi di sciogliere quesiti che rimarrebbero irrisolti avvalendosi unicamente delle competenze storico-artistiche. Ad esempio il riconoscimento di uno specifico pigmento ha permesso in molti casi di datare un'opera d'arte e di attribuirla ad uno specifico artista laddove lo studio puramente stilistico non lo consentiva. Inoltre lo studio archeometrico dei materiali (ovvero l'impiego della chimica analitica applicata ai beni culturali) permette di acquisire conoscenze inerenti la tecnologia impiegata nella realizzazione del manufatto.

Materiali quali la ceramica o il vetro hanno una grande valenza storico-artistica poiché sono stati ottenuti per la prima volta agli albori della civiltà e sono poi stati utilizzati in modo continuo fino ai nostri tempi accompagnando la storia delle civiltà. Tra questi materiali vi sono sicuramente il vetro, che secondo quanto tramandato da Plinio il Vecchio fu ottenuto per la prima volta in modo del tutto casuale lungo le sponde del fiume Belus (Libano) circa nel 3000 a.C., e la ceramica i cui primi reperti sembrano risalire al 26000 a.C. Le ceramiche e i vetri hanno accompagnato il cammino delle diverse civiltà trovando dapprima impiego come utensili ed oggetti di uso quotidiano (contenitori per la conservazione di sostanze solide o liquide) per poi essere utilizzati per la realizzazione di manufatti pregiati di grande valore artistico come nel caso delle maioliche rinascimentali italiane, decorate ed istoriate in maniera sublime.

Particolarmente interessante è il caso delle ceramiche rinascimentali italiane decorate con la tecnica del lustro, un film metallico iridescente di colore oro o rosso rubino ottenuto a partire da argento e da rame in forma nano-particellare mediante una tecnica molto sofisticata che prevedeva una terza cottura dell'oggetto in atmosfera riducente ed in condizioni 
estremamente controllate. Tale tecnologia testimonia le conoscenze estremamente avanzate di cui erano in possesso gli antichi vasai: la tecnica impiegata era così raffinata che ad oggi, nonostante i numerosi studi effettuati, il segreto dell'arte del lustro non è stato ancora completamente svelato e lo prova il fatto che i tentativi di imitazione di tali oggetti non eguagliano assolutamente le splendide maioliche del passato.

\section{IL LUSTRO DALL'ISLAM AL RINASCIMENTO: LA PRESENZA DEL BISMUTO NELLA PRODUZIONE ITALIANA}

Le ceramiche oggetto di questo studio sono maioliche lustrate caratterizzate dalla presenza di decorazioni a lustro, ovvero film dall'aspetto metallico iridescente applicati in terza cottura in atmosfera riducente ad oggetti precedentemente smaltati. Per quanto concerne la tecnologia con la quale venivano realizzate queste decorazioni si rimanda ad un contributo (Padeletti e Fermo, 2005) ad opera dei medesimi autori ed inserito nel presente volume.

I primi esemplari di maioliche lustrate vennero prodotte agli inizi del IX secolo d.C. in Mesopotamia, a Baghdad, centro del vasto impero arabo abbasside (A. Caiger-Smith, 1985). Successivamente, tra il X ed il XVI sec., in Persia, ed in particolare a Kashan, si sviluppò una fiorente industria per la produzione di ceramiche lustrate. In concomitanza quindi dell'espansione della cultura araba nel bacino del Mediterraneo, la tecnica del lustro venne sviluppata anche in Egitto, durante la dinastia fatimide. Alcuni studiosi sostengono che durante le insurrezioni che ebbero luogo in Mesopotamia (869-883), gli artigiani di Bosra, il secondo centro per importanza nella produzione ceramica in Iraq dopo Baghdad, in seguito ad una insurrezione che distrusse la città (871), migrarono verso l'allora nascente Egitto tulunide. Altri studiosi sostengono invece che le ceramiche ritrovate in Egitto e risalenti al IX sec. vennero direttamente importate dall'impero abbasside. In ogni caso tutti concordano sul fatto che i lustri prodotti a Samarra (Iraq) sono indistinguibili da quelli di epoca tulunide ritrovati in Egitto. Le più antiche ricette relative alla preparazione degli impasti per la produzione dei lustri islamici sono quelle tramandateci da Abu'l Qasim di Kashan (Iraq) (A. Caiger-Smith, 1985).

A partire dal XIII sec. i lustri cominciarono ad essere prodotti nella Spagna moresca che era stata conquistata dagli arabi cinquecento anni prima. La tecnica del lustro sopravvisse al crollo del mondo arabo avve- 
nuta nel XV sec. e gli artigiani ispano-moreschi fusero motivi di ispirazione araba e motivi tipicamente cristiani per creare maioliche che venivano esportate in Europa.

Numerose maioliche lustrate vennero quindi esportate dalla Spagna in Italia, Francia e Paesi Bassi. Il termine maiolica, utilizzato in Italia per indicare oggetti ceramici lustrati, trae infatti origine dall'isola di Majorca in Spagna. A quell'epoca le maioliche impreziosite con effetti metallici iridescenti atti ad imitare l'oro, erano molto ricercate dai mercanti italiani a causa del costo molto elevato degli oggetti realizzati con questo preziosa metallo. L'importazione delle maioliche lustrate dalla Spagna ebbe inizio circa trecento anni prima della comparsa dei primi esempi di lustri italiani.

A proposito dell' introduzione della tecnica in Italia sono state avanzate dagli studiosi alcune ipotesi. Durante il XV sec. i primi tentativi di imitazione di lustri ispano-moreschi ad opera di maestri italiani furono piuttosto infruttuosi sino a quando Galgano di Belforte apprese a Valencia il segreto dell'arte del lustro, gelosamente custodito dai maestri moreschi (Franchet, 1906) Poiché gli artigiani che operavano all'epoca a Faenza e a Firenze, i due maggiori centri di produzione ceramici, erano particolarmente abili, ed inoltre a causa dell'esistenza di numerosi contatti commerciali tra la Spagna e l'Italia, è improbabile che essi non conoscessero la tecnica del lustro. È invece possibile che, essendo già esperti nella produzione di ceramiche decorate di elevatissimo pregio, preferirono non cimentarsi nella realizzazione delle decorazioni a lustro (A. Caiger-Smith, 1985). La sperimentazione di questa nuova tecnica avvenne invece in piccoli centri di produzione ceramica, quali Gubbio e Deruta in Umbria, dove i motivi decorativi erano molto semplici e dove gli oggetti realizzati dagli artigiani erano destinati unicamente al mercato locale. Secondo quanto riportato da Caiger-Smith (A. Caiger-Smith, 1985), la tecnica del lustro venne importata a Deruta ad opera del famoso pittore Pinturicchio (1454-1413). Le ricette più dettagliate per la preparazione degli impasti da lustro che sono giunte sino a noi sono quelle riportate ne I tre libri dell'arte del vasaio ad opera di Cipriano Piccolpasso (Piccolpasso, 1557). Tali ricette, una per la realizzazione dei lustri oro ed una per la realizzaizone dei lustri rossi, erano quelle utilizzate nella bottega di Mastro Giorgio Andreoli (circa 1470-1555). Gli impasti erano costituiti da miscele di sostanze, tra le quali la terra rossa ed il ferretto di Spagna (CuS) (A. Caiger-Smith, 1985), con aggiunta di una moneta d'argento e di cinabro $(\mathrm{HgS})$ per l'ottenimento del lustro oro e l'aggiunta di bolo armeno per l'ottenimento del rosso. Tali miscele venivano poi disperse in aceto ad applicate ai manufatti. L'uso dell'aceto permetteva 
sia di mantenere la miscela allo stato flocculato consentendo di evitare la formazione di crepe in fase di essiccatura, sia di favorire la formazione di acetati ovvero sali facilmente decomponibili. Il lustro era quindi fissato in terza cottura a circa $600{ }^{\circ} \mathrm{C}$ in atmosfera riducente.

In virtù della loro abilità gli artigiani italiani svilupparono un loro proprio stile, anche se inizialmente si ispirarono ai lustri ispano-moreschi. Mentre a Deruta i ceramisti si specializzarono nella produzione dei lustri oro, Gubbio divenne famosa, grazie a Mastro Giorgio Andreoli, per la produzione di lustri dal tono rosso-rubino. E' importante sottolineare come il colore finale dipendesse non solo dalla composizione chimica dell'impasto ma anche dalle condizioni di cottura (Padeletti e Fermo, 2005). Il giallo oro, può infatti contenere notevoli quantità di rame che da luogo alla formazione di un lustro rosso-rubino in atmosfera riducente mentre, nelle prime fasi del processo di cottura quando l'atmosfera non è ancora completamente riducente, il colore che si ottiene è giallo-ambra.

A causa della preziosità dei manufatti lustrati e della difficoltà con cui essi venivano realizzati, le ricette per la preparazione degli impasti erano mantenute segrete. La qualità dei manufatti che vennero prodotti durante il Rinascimento non venne infatti mai più raggiunta durante il periodo di revival di questa tecnica nel corso del XIX sec. Oggi giorno la produzione di lustri simili a quelli antichi rappresenta per i ceramisti umbri una grossa sfida. Essi utilizzano generalmente composti di rame (ossido, acetato, cloruro o carbonato) per i lustri di colore rosso, composti a base di ferro (ocra, argille ferruginose o bolo armeno) per i lustri dai toni bronzei, cloruro di cobalto per il blu e nitrato di bismuto per ottenere effetti perlacei (Emiliani, 2001).

\section{CAMPIONI ANALIZZATI: DALLA PRODUZIONE ISLAMICA A QUELLA RINASCIMENTALE}

Oggetto di una prima fase del presente studio sono stati sia alcuni esempi di ceramiche rinascimentali lustrate di provenienza umbra, sia alcuni frammenti lustrati di tipo ispano-moresco rinvenuti in Umbria nel corso degli scavi. Tra i campioni italiani due frammenti sono stati identificati dagli esperti come imitazioni dello stile ispano-moresco. Una conferma dell'esistenza in Italia centrale di botteghe dove lo stile ispano-moresco veniva imitato e prodotto, è fornita dal ritrovamento in Umbria sia di frammenti originali ispano-moreschi che di imitazioni di tale stile, come 
nel caso dei frammenti esaminati in questo studio. In una seconda fase del lavoro sono stati inclusi nel confronto anche alcuni lustri islamici.

I campioni presi in esame sono databili al XV e al XVI sec e sono stati tutti rinvenuti in Umbria ad eccezione dei lustri islamici provenienti dall'Egitto ed attribuiti, sulla base di considerazioni stilistiche, al periodo fatimide (Fig. 1).
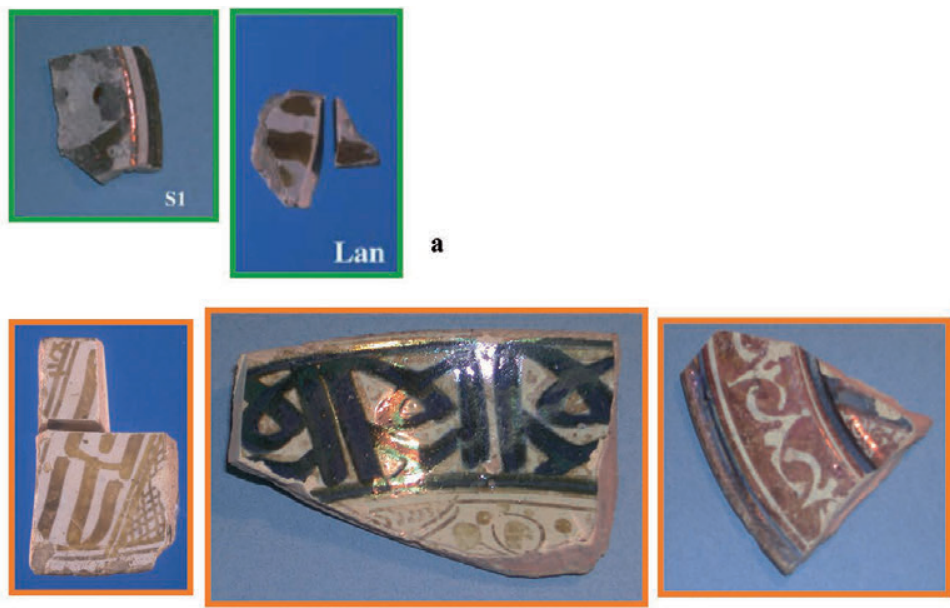

b
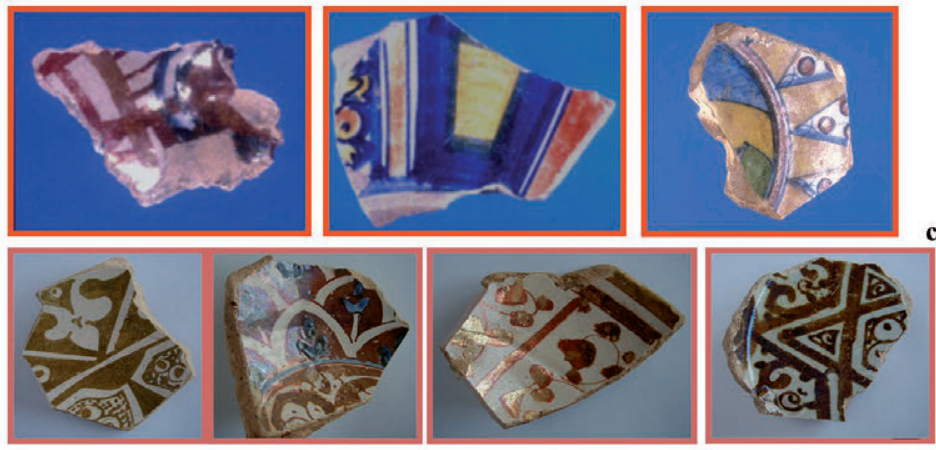

d

Fig. 1 - Alcuni esempi di frammenti di maioliche lustrate: a) imitazioni dello stile ispanomoresco; b) lustri ispano-moreschi; c) tipici lustri italiani; d) lustri islamici.

Essi includono sia lustri di colore oro che lustri di colore rosso decorati con motivi geometrici o floreali. Due lustri di colore oro (Lan e S1, Fig. 1a) sia a causa della peculiarità delle decorazioni che a causa del tono del lustro, sono stati definiti dagli esperti imitazioni dello stile ispano-moresco. Due dei campioni ispano moreschi (Fig. 1b) sono decorati con lustri oro mentre il terzo mostra un lustro dai toni rosso-ramati. 
L'analisi della composizione chimica del corpo ceramico dei frammenti è stata condotta prendendo in considerazione 8 elementi maggioritari ( $\mathrm{Ca}, \mathrm{Al}, \mathrm{Fe}, \mathrm{Mg}, \mathrm{K}, \mathrm{Ti}, \mathrm{Na}$ e $\mathrm{Mn}$ ) e 7 elementi minoritari (Cr, $\mathrm{Cu}$, $\mathrm{Ni}, \mathrm{Zn}, \mathrm{Zr}$, Sr e Rb). Le analisi sono state eseguite mediante ICP-OES (Inductively coupled plasma - optical emission spectrometry) sui campioni sottoposti a dissoluzione acida (in tabella 1 sono riportati i risultati relativi agli elementi maggioritari).

I risultati ottenuti sono stati quindi elaborati mediante metodi di calcolo chemiometrico [6]. Sulla base della composizione chimica è stato quindi possibile distinguere due gruppi di frammenti, uno costituito dai campioni italiani ed uno da quelli ispano.moreschi.

I metodi chemiometrici utilizzati sono stati la principal components analysis (PCA) e la cluster analysis (HCA). In Fig. 2 viene mostrato un dendrogramma (diagramma ad albero) nel quale i tre campioni ispanomoreschi formano un cluster a se stante mentre le due imitazioni rientrano in un cluster formato da campioni italiani. Si può quindi affermare i due campioni considerati imitazioni sono stati sicuramente prodotti in Italia centrale poiché presentano un corpo ceramico del tutto analogo chimicamente a quello dei campioni italiani. L'assegnazione effettuata puramente su basi stilistiche trova quindi una conferma scientifica.

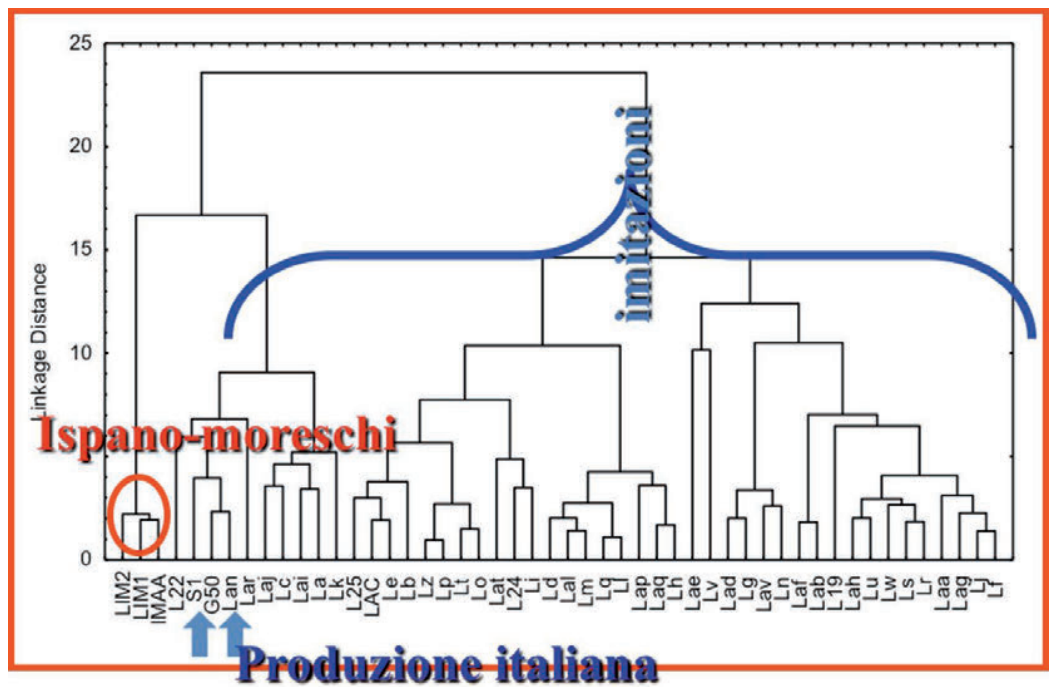

Fig. 2 - Dendrogramma ottenuto dalla cluster analysis eseguita sui dati di composizione chimica relativi a ceramiche lustrate italiane ed ispano.moresche. 
La presenza di almeno tre grossi cluster all'interno della produzione italiana è legata a differenze minori nella composizione chimica dei materiali di partenza.

Una ulteriore prova a favore dell'attribuzione italiana dei due frammenti definiti imitazioni, è fornita dall'esame dello smalto (Fig. 3): infatti, analogamente ai campioni italiani, le due imitazioni mostrano uno smalto avente spessore mag giore di quello tipico delle ceramiche ispano-moresche.

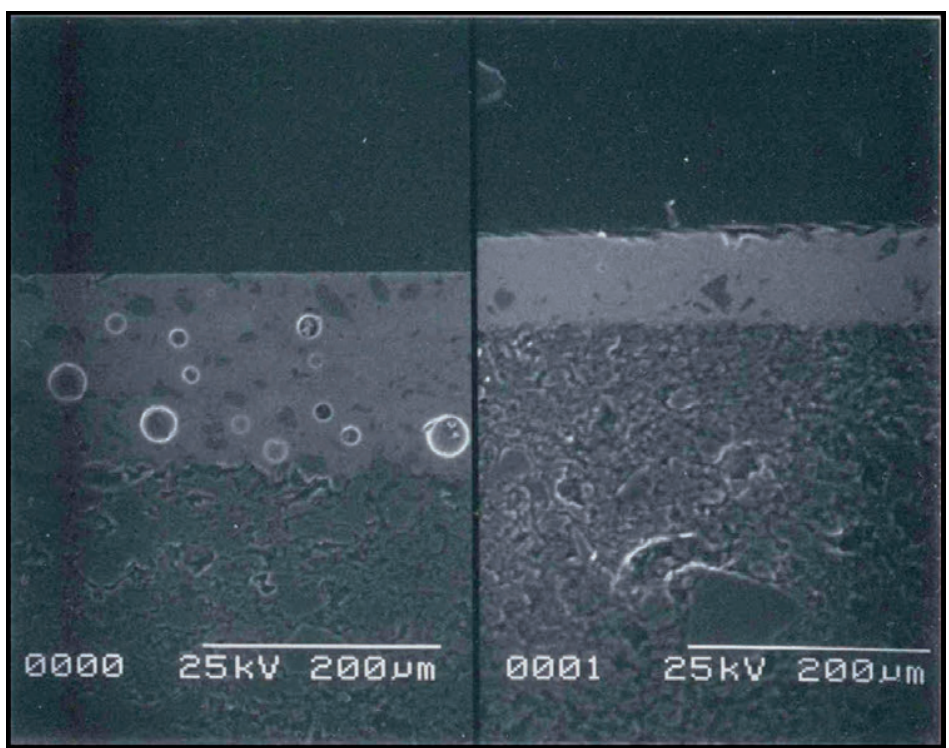

Fig. 3 - Immagini ottenute mediante SEM (scanning electron microscopy) ed acquisite rispettivamente su un campione definito imitazione dello stile ispano-moresco ed su un campione ispano moresco.

Di fondamentale importanza ai fini della caratterizzazione dei lustri si è rivelata essere l'analisi XRD grazie alla quale è emersa una importante differenza tra la produzione italiana e quella ispano-moresca. Infatti i diffrattogrammi acquisiti sui campioni di lustro italiani mostrano la presenza di un segnale dovuto alla cosalite, una fase cristallina corrispondente alla formula $\mathrm{Pb}_{2} \mathrm{Bi}_{2} \mathrm{~S}_{5}$ che non è presente nei campioni ispano-moreschi. Nel caso delle due imitazioni questa fase è presente ma il segnale è poco intenso. Come è noto la cosalite è una fase che si può formare quando il bismuto è presente unitamente a piombo e zolfo (Anthony, 1990). È importante sottolineare che la presenza di 
bismuto all'interno dei lustri non era mai stata evidenziata sino ad ora. $\mathrm{Al}$ fine di confermare la presenza di questo elemento, che non compare come ingrediente in nessuna delle ricette giunte sino a noi, sono state condotte analisi mediante la tecnica ETAAS (electrothermal atomization absorption spectroscopy), ovvero una tecnica di analisi elementare in grado però di raggiungere limiti di rilevabilità più bassi rispetto all'ICP-OES. Unitamente al bismuto sono stati quantificati anche il rame e l'argento. I rapporti $\mathrm{Bi} / \mathrm{Ag}$ sono riportati in tabella 3 e variano all'interno del range 0.42-5.6 indicando che la quantità di bismuto addizionata all'impasto dai vasai era piuttosto variabile ed in molti casi superiore alla quantità di argento. Mentre il bismuto è stato rilevato in tutti i campioni italiani, esso è risultato essere assente nei campioni ispano-moreschi e nelle due imitazioni (in questo secondo caso la concentrazione presente era però al di sotto del limite di rilevabilità).

Anche i rapporti $\mathrm{Cu} / \mathrm{Ag}$ sono piuttosto variabili e vanno da 90 a 0.1 per i lustri oro e da 33 a 4 nel caso dei lustri rossi. Inoltre alcuni lustri rossi, incluso il campione ispano-moresco, non contengo no argento mentre alcuni lustri oro italiani non contengono rame. Del resto è già stato dimostrato (Padeletti e Fermo, 2005) come il colore non dipenda unicamente dalla composizione chimica ma sia anche legato alle condizioni di cottura. Poiché il rame, a differenza dell'argento e del bismuto, può essere presente come elemento minoritario anche all'interno dello smalto, per poter quantificare il rame nel lustro ne è stata quantificata la concentrazione nello smalto ed è stata quindi effettuata una sottrazione (Padeletti e Fermo, 2003). A causa dell'esiguo spessore del lustro le scaglie sottoposte a dissoluzione sono infatti mediamente costituite per il $99 \%$ da smalto e per l'1\% dal lustro.

Mentre rame ed argento sono stati evidenziati innanzitutto mediante analisi SEM-EDX (scanning electron microscopy - energy dispersion $\mathrm{X}$ ray spectroscopy) (Padeletti e Fermo, 2005) e successivamente quantificati tramite ETAAS, la presenza del bismuto non è evidenziabile mediante EDX a causa della sovrapposizione con il segnale del piombo, presente in concentrazione molto elevata nello smalto sottostante il lustro. Di conseguenza la diffrazione di raggi $\mathrm{X}$ si è rivelata fondamentale per l'individuazione del bismuto. Questo elemento, come già ricordato, non compariva tra gli ingredienti per la preparazione degli impasti da lustro. Tuttavia, Cipriano Piccolpasso (Piccolpasso, 1557) afferma che egli non assistette personalmente alla preparazione degli impasti ma semplicemente ne senti parlare poiché le ricette venivano gelosamente custo- 
dite e mantenute segrete. Pertanto le ricette che egli trascrisse molto probabilmente differiscono da quelle realmente utilizzate.

Per poter spiegare la presenza di questo elemento nello strato di lustro è stata presa anche in considerazione la possibilità che il bismuto venisse introdotto come impurezza dell'argento, inserito nell'impasto sotto forma di moneta (carlino d'argento). Il bismuto potrebbe infatti essere presente come impurezza, pari al massimo allo 0.1\% (Giardino, 1998), nel minerale grezzo contenente argento ed impiegato per la produzione delle monete. In questo caso la presenza del bismuto negli impasti non sarebbe quindi intenzionale. Al fine di verificare questa ipotesi, si è proceduto con l'analisi di alcune monete coniate in epoca rinascimentale e sono stati quantificati sia il bismuto che l'argento. All'interno delle monete la concentrazione massima di bismuto riscontrata è stata pari a $0.157 \%$, ovvero piuttosto bassa. Se il bismuto fosse stato introdotto come impurezza della moneta non sarebbe quindi rilevabile a causa della concentrazione troppo bassa che risulterebbe inferiore al limite di rilevabilità della tecnica per questo elemento. La tecnica XRD interessa l'analisi del solo strato superficiale, a differenza dell'ETAAS che analizza l'intero spessore costituito dal lustro e dallo smalto e per tale ragione l'XRD può essere considerata più sensibile. $\mathrm{Nel}$ caso dei due lustri identificati come imitazioni la cosalite è stata infatti identificata mediante XRD; tuttavia questa fase è presente in concentrazione piuttosto bassa ed il bismuto non è stato identificato.

È pertanto possibile concludere che la bassa percentuale di bismuto presente nelle monete fa sì che tale elemento non risulterebbe rilevabile mediante ETAAS. Si può quindi escludere che nei lustri il bismuto sia presente come impurezza della moneta d'argento.

Un aspetto molto interessante da prendere in considerazione è rappresentato dalle notizie storiche riguardanti la conoscenza del bismuto come elemento durante il Rinascimento. Agli inizi del XVI sec. il bismuto era infatti già noto in Europa quale elemento distinto. Esso viene infatti menzionato a proposito dell'uso che se ne faceva in alcune pitture medievali dove lo strato preparatorio era costituito da bismuto (tecnica risalente al XV sec.) (Lippmann, 1930). Inoltre, come viene riportato da Agricola (1494-1555) (Bauer) (disponibili presso la Biblioteca Nazionale Braidense, Milano), il pioniere delle scienze mineralogiche in Europa, il bismuto era considerato un elemento differente da quelli noti all'epoca e cioè argento, rame, ferro, oro, piombo e sta- 
gno. Infatti il bismuto era detto "piombo ceneraccio" mentre il piombo e lo stagno erano chiamati rispettivamente "piombo nero" e piombo bianco". Il bismuto era anche chiamato "testum argenti" poiché si supponeva si trattasse di argento da dalla natura imperfetta.

Il bismuto era presente nelle miniere della Boemia e della Sassonia e la sua presenza indicava che al di sotto dello strato superficiale costituito da questo elemento era possibile trovare l'argento. Quindi in quell'epoca il bismuto poteva essere confuso con l'argento da chi non era molto esperto. $\grave{E}$ interessante osservare come la maggior parte delle miniere dalle quali può essere estratto il bismuto sono concentrate in Europa, in Africa sono presenti solo in Rodesia mentre in nord Africa ed in Asia Minore sono completamente assenti (Fig. 4) (Mellor, 1929). Lo stesso Agricola afferma che mentre il bismuto era noto in Europa, esso era sconosciuto al mondo arabo (Partington, 1961).

A testimonianza di quanto fosse intrigante la storia del bismuto vi è un'opera del tedesco Von Lippmann che nel 1930 scrisse addirittura un trattato sulla storia del bismuto tra il 1400 ed il 1800 (Lippmann, 1930).

Il bismuto in Europa veniva anche utilizzato unitamente a piombo e stagno per produrre vasi, piatti, ciotole e boccali. Infatti Entzel (1520-1586) nel suo scritto dedicato ai metalli ed alle pietre (come viene riportato da Partington) sostiene che questo metallo venisse utilizzato per contraffare ed imitare i vasi d'argento. Anche Entzel afferma che il bismuto era sconosciuto al mondo arabo.

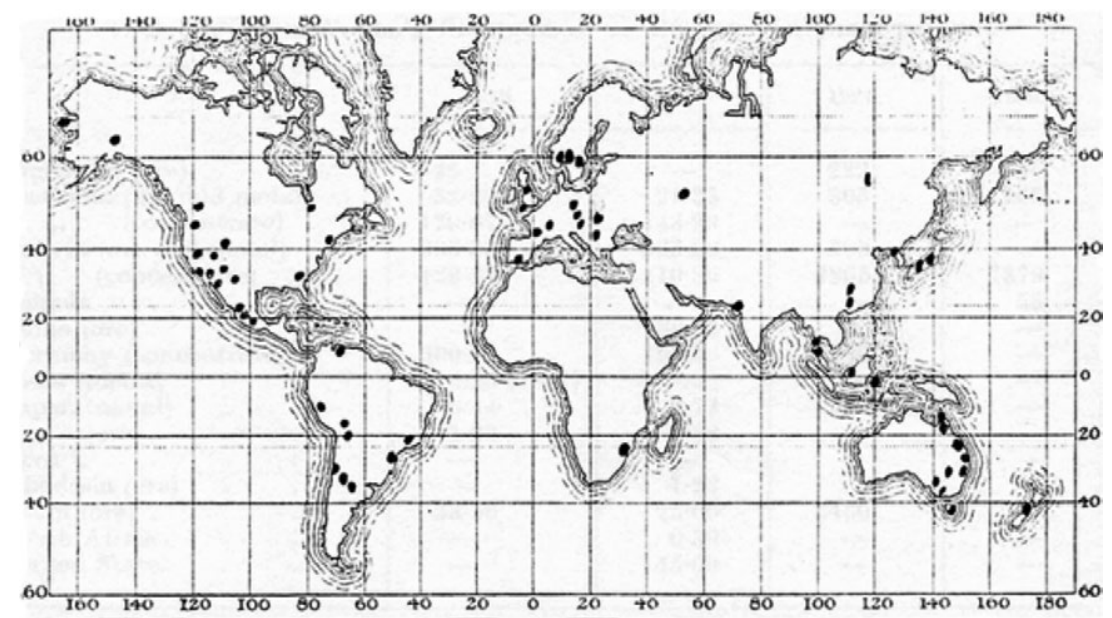

Fig. 4 - Distribuzione dei giacimenti di bismuto. 
La conoscenza del bismuto da parte degli artigiani italiani è confermata da Franchet [3] che tentò di riprodurre i lustri rinascimentali agli inizi del XX sec.

Poiché il bismuto non era quindi noto nel mondo islamico e poiché le ricette ispano-moresche vennero direttamente importate dall'Islam, l'assenza di questo elemento nei lustri islamici è quindi giustificata.

$\mathrm{Al}$ contrario i ceramisti italiani, anche se appresero la ricetta dagli spagnoli, furono in grado di sviluppare ricette proprie ed originali stili decorativi introduc per tale ragione endo notevoli variazioni. Probabilmente all'epoca in Italia l'uso del bismuto al fine di imitare l'argento ero noto e non si può pertanto escludere che gli artigiani aggiungessero questo elemento all'impasto da lustro con lo scopo di contenerne il costo. Inoltre è anche probabile che essi sapessero che il bismuto non era noto agli arabi e questo potrebbe spiegare le piccole quantità di cosalite riscontrate nelle imitazioni dello stile ispano-moresco.

Un ulteriore motivo che giustificherebbe l'uso del bismuto potrebbe risiedere nel fatto che questo elemento, addizionato all'impasto, contribuisce ad abbassare considerevolmente le temperature di fusione sia del rame che dell'argento, come può essere osservato nei rispettivi diagrammi di fase. E' anche interessante osservare come alcuni composti del bismuto (ossido, carbonato e nitrato) vengano utilizzati per la preparazione di lustri moderni (A. Caiger-Smith, 1985). Questi composti agiscono come fondenti nei cosiddetti "pigmenti attivi": essi consentono cioè al pigmento di aderire allo smalto ad una temperature sufficientemente bassa da evitare che lo smalto scolori in seguito al processo di riduzione dell'ossido di piombo a piombo metallico. Inoltre l'ossido di bismuto può anche conferire una tonalità perlacea al lustro stesso (A. Caiger-Smith, 1985).

Tra la produzione italiana a lustro e quella ispano-moresca vi è una importante differenza dal punto di vista della composizione chimica dello strato di lustro. Questa differenza, messa in evidenza tramite diffrazione di raggi $\mathrm{X}$ ed analisi elementare, è legata alla presenza del bismuto unicamente nei lustri italiani. Tale elemento, presente come cosalite, $\mathrm{Pb}_{2} \mathrm{Bi}_{2} \mathrm{~S}_{5}$, un solfuro misto di piombo e bismuto che si forma durante il processo di cottura del lustro, può quindi essere considerato un marker distintivo della produzione italiana.

Poiché in epoca rinascimentale in Italia era probabilmente noto l'uso del bismuto per imitare l'argento, non si può escludere che i cera- 
misti italiani aggiungessero intenzionalmente questo elemento per contenere il costo dell'impasto da lustro. Inoltre questo elemento non era noto nel mondo arabo e questo potrebbe spiegare per quale motivo esso sia assente nei lustri ispano-moreschi (preparati secondo ricette direttamente importate dall'Islam) e presente invece in piccole concentrazioni nelle imitazioni dello stile ispano-moresco rinvenute in Umbria.

Un ulteriore importante differenza tra le produzioni italiana e spagnola è stata riscontrata nella composizione del corpo ceramico. Tale differenza è stata evidenziata grazie all'analisi elementare dei corpi ceramici e alla successiva elaborazione dei dati mediante metodi di calcolo multivariato. Entrambe questi metodi hanno messo in evidenza la presenza di due gruppi distinti, uno formato dai campioni italiani e uno da quelli ispano-moreschi. Grazie a questo tipo di approccio è anche emerso come le imitazioni ispano-moresche siano state realizzate a partire da maioliche di produzione italiana decorate però mediante un impasto da lustro preparato secondo la ricetta spagnola. L'assenza di bismuto è stata riscontrata anche all'interno dei lustri islamici ed è una prerogativa sia dei lustri di produzione irachena che di quelli fatimidi a riprova del fatto che il bismuto non fosse noto nel mondo arabo.

\section{BIBLIOGRAFIA}

G. Padeletti, P. Fermo. Studio di frammenti di maioliche rinascimentali lustrate: principali risultati, Simposio internazionale Mastro Giorgio da Gubbio: Arte, Scienze e Tecnologia delle Maioliche a Lustro, 9-11 Novembre 2005, Gubbio (Italy).

A. Caiger-Smith. Luster Pottery: Technique, Tradition and Innovation in Islam and the Western World (Faber and Faber, London 1985), p. 197.

M.L. Franchet. Ann. Chem. Phys. Ser. 8, IX, (1906).

C. Piccolpasso. Li Tre Libri dell'Arte del Vasaio, 1557 (Edizioni all'insegna del Giglio, Firenze 1976).

G.P. Emiliani, F. Corsara. Tecnologia Ceramica, la Lavorazione, Vol. II (gruppo editoriale Faenza editrice, Faenza, Italy 2001).

G. Pdeletti, P. Fermo. Applied Physics A 77 (2003) 125-133.

J.W. Anthony, R. Bideaux, K.W. Bladth, M.C. Nicols. Handbook of Mineralogy, Vol. I, Element Sulpbides and Sulfo-salts (Mineralogical Data, Tucson, AZ 1990).

C. Giardino. I metalli nel mondo antico. Introduzione all'archeometallurgia (Laterza and Figli, Roma 1998).

J.R. Partington. A History of Chemistry, Vol. 2 (Macmillian, London 1961).

E.O. von Lippmann. Die Geschichte des Wismuts zwischen 1400 und 1800: ein Beitrag zur Geschichte der Technologie und der Kultur (Springer, Berlin 1930). 
G. Bauer or G. Agricola. De Natura Fossilium, Lib. X (Froben, Basel 1546).

G. Bauer or G. Agricola. Bermannus Sive De Re Metallica Dialogus (Froben, Basel 1528).

G. Bauer or G. Agricola. Bermannus Sive De Re Metallica Dialogus (Froben, Basel 1546).

J.M. Mellor. A Comprehensive Treatise on Inorganic and Theoretical Chemistry, Vol. IX (Longman Green, London 1929) p. 587. 